\title{
Nachfrage und Auslastung von Fortbildungen für Lehrerinnen und Lehrer: Effekte struktureller und inhaltlicher Angebotsmerkmale
}

\author{
Karen Johannmeyer (D) Colin Cramer $\mathbb{D}$
}

Eingegangen: 5. Dezember 2019 / Überarbeitet: 10. März 2021 / Angenommen: 25. März 2021 / Online publiziert: 1. September 2021

(C) Der/die Autor(en) 2021

Zusammenfassung Fortbildungen kommt in der berufslebenslangen Professionalisierung von Lehrpersonen eine wichtige Funktion zu. Bislang wurde primär zu deren Wirksamkeit oder zu Merkmalen der Teilnehmenden geforscht. Das Angebot an Fortbildungen wurde kaum untersucht und steht im Fokus dieser Studie: Strukturelle sowie inhaltliche Merkmale der Fortbildungen werden beschrieben und empirische Zusammenhänge mit deren Nachfrage und Auslastung hergestellt. Die Datengrundlage bilden Ausschreibungen von $N=6240$ regionalen, schulexternen Fortbildungen, die im Zeitraum eines Jahres unter Verantwortung des Kultusministeriums in BadenWürttemberg angeboten wurden. Ausgehend von inhaltsanalytischen Kodierungen der Angebotsbeschreibungen wird regressionsanalytisch gezeigt, dass eine höhere Nachfrage sowie Auslastung nicht nur durch die Dauer der Fortbildungen, sondern auch durch deren Fachbezug sowie deren Inhalte erklärt werden können. Implikationen für die Fortbildungsplanung und für weitere Forschung werden diskutiert.

Schlüsselwörter Lehrerbildung · Lehrerfortbildung · Angebot · Nachfrage

Karen Johannmeyer $(\varangle) \cdot$ Prof. Dr. Colin Cramer $(\varangle)$

Tübingen School of Education, Universität Tübingen, Wilhelmstraße 31, 72074 Tübingen,

Deutschland

E-Mail: karen.johannmeyer@uni-tuebingen.de; colin.cramer@uni-tuebingen.de 


\title{
Demand for and participation rate of professional development courses for teachers: effects of structural and content-related characteristics of the courses
}

\begin{abstract}
Continuing education plays an important role in the professionalization of teachers. Whereas past studies have focused on the effectiveness of professional development programs, little attention has been paid to the structure and content of the programs themselves. In this study structural and content-related features of professional development courses are described, and empirical correlations with demand and participation rate are established. The analyses are based on a dataset with descriptions of $N=6240$ professional development courses offered for all teachers during the course of one year under the responsibility of the ministry of education and cultural affairs in Baden-Wuerttemberg, Germany. Based on a content analysis of the course descriptions, a regression analysis shows that a higher demand and participation rate can not only be explained by the duration of the courses, but also by the subjects and the topics they address. Lastly, possible implications for the planning of professional development courses and for further research are discussed.
\end{abstract}

Keywords Demand · Participation · Professional development · Teacher education

\section{Einleitung}

Fortbildung wird eine wichtige Funktion für die berufslebenslange Professionalisierung von Lehrerinnen und Lehrern zugeschrieben (Böttcher et al. 2019; Pasternack et al. 2017). Sie trägt dazu bei, den unterschiedlichen und sich stets wandelnden Herausforderungen durch einen kontinuierlichen Prozess der Professionalisierung zu begegnen (Fabel-Lamla 2018). In der empirischen Forschung wurde die Nutzung von Fortbildungen als Anlass der Professionalisierung vor allem im Zusammenhang mit Merkmalen von Teilnehmenden untersucht (z. B. Richter et al. 2011; Richter 2016) oder es wurde gefragt, in welchem Umfang welche Fortbildungsangebote genutzt werden (z.B. Hoffmann und Richter 2016). Das Angebot an Fortbildungen stand bislang vor allem im Kontext der Frage nach Merkmalen erfolgreicher, d.h. ,wirksamer ' Fortbildungen zur Diskussion, wobei in längsschnittlichen Primärstudien die Wirksamkeit von Fortbildungen bis auf die Ebene des Lernens von Schülerinnen und Schülern untersucht wurde (z. B. Garet et al. 2001). In (systematischen) Literaturreviews wurden strukturelle und inhaltliche Merkmale sowie Aktivitäten in der Fortbildung auf deren Wirksamkeit untersucht (Darling-Hammond et al. 2017; Desimone 2009; Yoon et al. 2007).

Weitestgehend unberücksichtigt bleibt bislang die Frage, welche Fortbildungsangebote in der Breite überhaupt ausgebracht werden und inwiefern die Nachfrage dieser Angebote mit den Merkmalen der Fortbildungen zusammenhängt. In der Wirksamkeitsforschung wurden bisher primär Fortbildungen in den Blick genommen, die einen Fokus auf Didaktik und Unterricht legen. Da jedoch auch umfangreich Fortbildungen zu anderen Themen ausgebracht werden (vgl. für Baden-Württemberg: Johannmeyer et al. 2019b), ist zu fragen, wie sich die Nachfrage zu unterschiedli- 
chen Themen gestaltet und ob auch strukturelle Merkmale, wie beispielsweise der Zeitpunkt der Fortbildungen, Einfluss auf die Nachfrage haben.

Eine bislang kaum genutzte Möglichkeit, Angebotsmerkmale in die Forschung nach dem Nutzungsverhalten mit aufzunehmen, ist durch Programmanalysen gegeben (Käpplinger 2011; Nolda 2018), in denen auf Grundlage von Datenbanken das Fortbildungsangebot analysiert wird. Erste Studien nehmen z. B. die Gesamtstruktur des Angebots in den Blick (Johannmeyer et al. 2019b) oder untersuchen die Anzahl der Teilnehmenden im Zusammenhang mit zeitlichen Merkmalen des Fortbildungsbesuchs (Richter et al. 2020). Die vorliegende Studie erweitert solche Forschung, indem multiple strukturelle und inhaltliche Merkmale von Fortbildungen als Prädiktoren der Nachfrage modelliert werden. Damit soll ein Beitrag zur Beantwortung der zentralen Forschungsfrage geleistet werden, welche Arten von Fortbildungsangeboten von Lehrerinnen und Lehrern besonders stark nachgefragt werden und in welchem Ausmaß das vorgehaltene Angebot mit der Nachfrage korrespondiert.

\section{Rahmenmodell, Stand der Forschung und Fragestellungen}

Als Ausgangspunkt wird ein Rahmenmodell zur Wirksamkeit von Fortbildungen für Lehrpersonen eingeführt (2.1). Dann werden auf Basis des einschlägigen Forschungsstandes personengebundene, strukturelle und inhaltliche Merkmale der Fortbildungen als bedeutsam für das Fortbildungsverhalten von Lehrpersonen herausgestellt (2.2) und schließlich die Fragestellungen für die empirische Studie konkretisiert (2.3).

\subsection{Rahmenmodell: Angebot und Nutzung von Fortbildungen}

Ein Rahmenmodell zur Erklärung der Wirksamkeit von Fortbildungen wurde von Lipowsky (2010) in Anlehnung an Angebots-Nutzungs-Modelle aus der Unterrichtsforschung formuliert (vgl. Abb. 1). Das Fortbildungsangebot lässt sich dem Modell zufolge mit Blick auf seine Ziele und Konzeption durch strukturelle und inhaltliche Merkmale, durch die Aktivitäten innerhalb der Fortbildung sowie durch die Expertise der Fortbildenden beschreiben. Die Wahrnehmung und Nutzung der Angebote wird durch personengebundene Voraussetzungen der Teilnehmenden beeinflusst, die wiederum den Transferprozess und damit den Fortbildungserfolg beeinflussen. Die Einbettung von Individuum sowie Bildungsinstitution in allgemeine Organisationsstrukturen und Regelungen wird im Mehrebenenmodell von Boeren, Nicaise und Baert (2010) betont: Eine Interaktion zwischen Individuum und Bildungsinstitution und damit auch die wechselseitige Beeinflussung von Angebot und Nachfrage (Nutzung) wird fokussiert. Im Modell von Lipowsky (2010) sind Kontextbedingungen (schulische Anforderungen usw.) zwar enthalten, strukturelle Rahmenbedingungen des Systems (z. B. Organisationsstruktur oder Gesetzgebung) werden aber nicht explizit berücksichtigt und daher in Abb. 1 ergänzt.

Konform zu gesetzlichen Grundlagen gibt es zwar in allen Bundesländern eine Pflicht zur Fortbildung, allerdings nur in Bayern, Bremen und Hamburg konkrete Vorgaben zum zeitlichen Umfang der zu besuchenden Angebote. Nur in Hamburg 


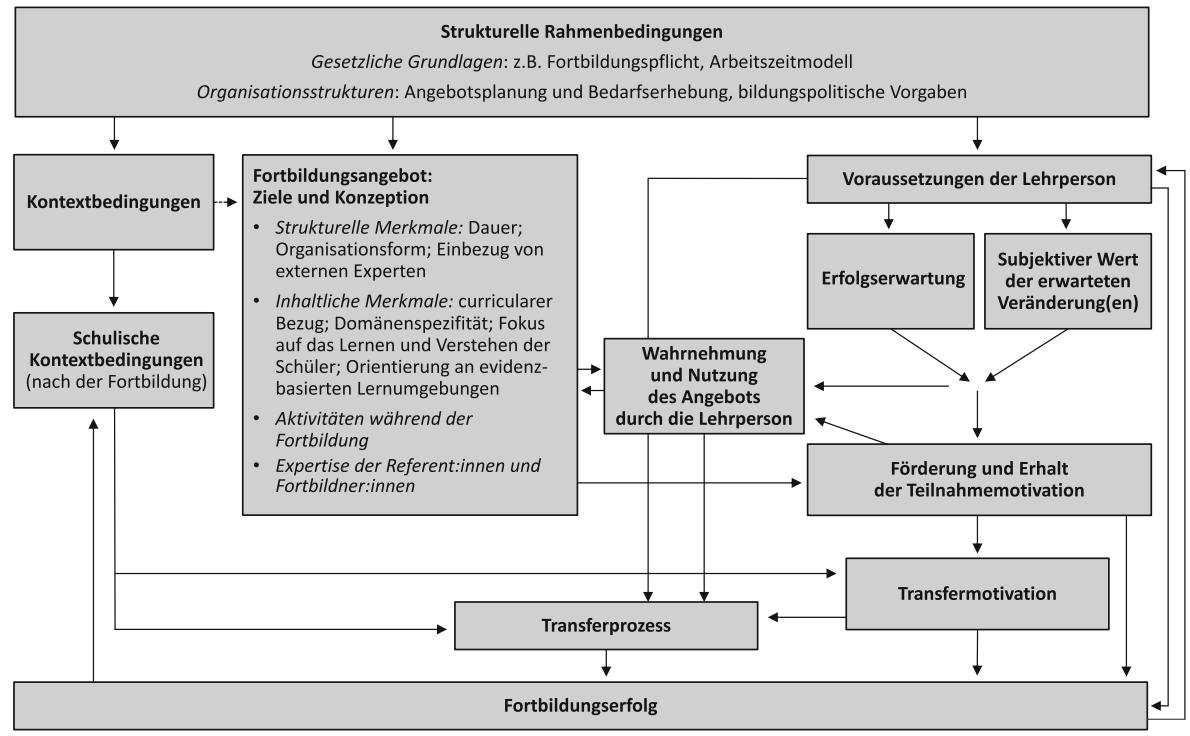

Abb. 1 Erweitertes Rahmenmodell der Wirksamkeit von Fortbildungen für Lehrerinnen und Lehrer (in Anlehnung an Lipowsky 2010, S. 51)

werden Fortbildungszeiten auch explizit im Arbeitszeitmodell und bei der Berechnung des Lehrpersonenstellenbedarfs berücksichtigt (Daschner und Hanisch 2019, S. 24). Da die Arbeitszeitmodelle in der Regel auf der Definition von Deputatsstunden beruhen, finden die Fortbildungen in den anderen Bundesländern meist außerhalb der Unterrichtszeit statt, oder Unterricht muss vertreten werden oder entfallen (Daschner und Hanisch 2019, S. 24).

Durch Organisationsstrukturen wird über Bedarfserhebung oder Angebotsplanung das Fortbildungsangebot beeinflusst. Die Fortbildung von Lehrpersonen ist in den deutschen Bundesländern in der Regel kaskadenförmig organisiert (Daschner und Hanisch 2019, S. 22 ff.): Ziele, Inhalte, Schwerpunkte und Rahmenbedingungen werden zum Teil ministeriell festgelegt und von staatlichen Einrichtungen umgesetzt. Teilweise geht der Angebotsplanung auch eine Bedarfserhebung voraus, die von den verschiedenen Institutionen je unterschiedlich durchgeführt wird und von standardisierten Befragungen bis hin zu informellen individuellen Gesprächen reicht (Johannmeyer et al. 2019a). Da der erhobene Bedarf so bereits die Angebotsplanung beeinflusst, zeigt sich eine durch die Organisationsstrukturen bedingte Interdependenz von Angebot und Wahrnehmung des Angebots durch die Lehrpersonen.

\subsection{Empirische Befunde zu Angebot und/oder Nutzung von Fortbildungen}

Im Folgenden werden empirische Befunde zum Verhältnis von Angebot und Nutzung von Fortbildungen vorgestellt. Da eine Programmanalyse (Nolda 2018) vorgelegt wird, bei der die Relevanz von Angebotsmerkmalen für die Nachfrage im Mittelpunkt steht und die Ausschreibungen der Fortbildungen als Datengrundlage dienen, sind Befunde zu strukturellen (z.B. Dauer oder Organisationsform des Angebots) 
sowie inhaltlichen Merkmalen (z. B. curricularer Bezug und Domänenspezifität) von besonderer Bedeutung und werden umfassender dargestellt.

Eine wichtige Referenzgröße für die Frage nach Prädiktoren des Nutzungsverhaltens insgesamt stellen personengebundene Merkmale dar. Es zeigt sich in der empirischen Forschung der im Rahmenmodell angenommene Zusammenhang dieser Merkmale mit der Nutzung der Angebote: So wird ein Zusammenhang zwischen einer vergleichsweise hohen Selbstwirksamkeit und einem häufigeren Besuch von Fortbildungen berichtet (Richter et al. 2013, S. 202). Auf Basis der ,Selbstbestimmungstheorie der Motivation " wird über das Konstrukt der ,Fortbildungsmotivation “ nach Gründen für die Fortbildungsteilnahme geforscht (Gorozidis und Papaioannou 2014; Kao et al. 2011; Richter et al. 2019; Rzejak et al. 2014). Als häufigste Gründe, nicht an Fortbildungen teilzunehmen, werden von Lehrpersonen eine als mangelhaft wahrgenommene Qualität der Angebote (Richter et al. 2018) sowie die Unvereinbarkeit mit den Arbeitszeiten (OECD 2014; Richter et al. 2018) genannt.

Die Nutzung von Fortbildungen wird auch durch die Wahrnehmung der Angebote beeinflusst: Während sich einige Lehrpersonen z. B. zum Informationsgehalt der Ausschreibungen zufrieden äußern (Haenisch 1992; Jesch und Keil 2009), werden diese von anderen als zu wenig detailliert und als lückenhaft angesehen, und es werden weitere Informationen z. B. zu Inhalten, Zielen, dem Ablauf der Veranstaltung oder zur Expertise der Dozierenden gewünscht (Haenisch 1992; Wolf et al. 1997). Lehrpersonen nehmen die Sichtung der Angebote im Schulalltag als zusätzlichen Aufwand wahr, was eine Teilnahmehürde darstellen kann (Richter und Vigerske 2011).

\subsubsection{Strukturelle Merkmale}

Richter et al. (2020) untersuchen zeitliche Merkmale von Fortbildungsangeboten im Zusammenhang mit deren Nutzung. Fortbildungen unter 2 bzw. über $8 \mathrm{~h}$ Dauer haben weniger Teilnehmende, während Angebote zwischen 2 und $8 \mathrm{~h}$ Dauer sowie Fortbildungsreihen eine höhere absolute Zahl an Teilnehmenden aufweisen. Ebenso zeigt sich eine höhere Teilnehmendenzahl bei Beginn am Vormittag. Diese könnte sich jedoch auch dadurch erklären lassen, dass hier am Vormittag weitaus weniger Veranstaltungen stattfinden als am Nachmittag: Dass Fortbildungen ggf. um Teilnehmende konkurrieren und die Nachfrage somit im Zusammenhang mit dem Angebot steht, findet keine Berücksichtigung. Die abhängige Variable der Teilnehmendenzahl alleine lässt zudem die Frage offen, ob es über die verfügbaren Teilnahmeplätze hinaus eine weitere Nachfrage gegeben hat, oder ob weniger Nachfrage bestand als Plätze vorgesehen waren.

Es existiert unseres Wissens keine Forschung dazu, inwiefern die Schulform, für die ein Angebot ausgeschrieben ist (strukturelles Merkmal), in einem Zusammenhang mit der Nachfrage steht. Eine Annäherung ist über die Betrachtung der Nutzung differenziert nach der Schulform der Teilnehmenden (personengebundenes Merkmal) möglich: Lehrpersonen aus Schulen mit mehreren Bildungsgängen besuchen häufiger Fortbildungen in der Breite der Themen, an der Hauptschule hingegen öfter Fortbildungen zu pädagogischen Fragestellungen, wie z. B. zur Klassenführung (Richter et al. 2013). Bezüglich der Anzahl der besuchten Fortbildungen insgesamt 
zeigen sich allerdings keine signifikanten Unterschiede zwischen Lehrpersonen verschiedener Schulformen (Richter 2011).

\subsubsection{Inhaltliche Merkmale}

Zur Nachfrage an Fortbildungen für bestimmte Schulfächer liegen bislang vergleichsweise wenige empirische Ergebnisse vor. Dies lässt sich durch teils sehr spezifische fachbezogene Forschung erklären, die sich häufig auf die Wirksamkeit spezifischer Angebote bezieht (z.B. für Mathematik: Schultis et al. 2014). Auch basiert die Forschung häufig auf Stichproben, die auf ein Fach begrenzt sind (z. B. Mathematik in der COACTIV-Studie, Kunter et al. 2011; wechselnde Fächerschwerpunkte im IQB-Bildungstrend, z. B. Pant et al. 2013; Stanat et al. 2016), was einen Fachvergleich der Nutzung einschränkt. Mathematiklehrpersonen geben an, etwas häufiger fachspezifische als allgemeine Fortbildungen zu besuchen (Jäger und Bodensohn 2007). In Baden-Württemberg interessieren sich Lehrpersonen offenbar vermehrt für fachliche (im Sinne von fachbezogenen) Fortbildungen (Ministerium für Kultus, Jugend und Sport Baden-Württemberg 2017). Dies spiegelt sich nicht im Angebot wider: etwa die Hälfte der Fortbildungen weist keinen expliziten Fachbezug auf (Johannmeyer et al. 2019b, S. 48).

Die Themen, zu denen Fortbildungsbedarf besteht, wurden international untersucht: Im OECD-Durchschnitt artikuliert ein Drittel der Befragten einen Bedarf an Fortbildungen zum Unterrichten von Schülerinnen und Schülern mit besonderen Lernbedürfnissen, ein Viertel zum Umgang mit Computer und Internet und ein Fünftel zeigt Interesse an Fortbildungen zu Fragen nach Disziplin und Verhalten (OECD 2009, S. 60 f.). In einer korrespondierenden Studie für den deutschsprachigen Raum wird der größte Fortbildungsbedarf zu den Themen ,Unterrichten von Schülern mit besonderen Lernbedürfnissen“ (42\%) und ,Probleme mit Schülerinnenund Schüler-Disziplin und Verhalten' (33\%) berichtet (Gargarina und von Saldern 2010, S. 54). Richter et al. (2013) identifizieren fünf Gruppen mit differenziellen Profilen: Lehrpersonen, die Fortbildungen zu allen genannten Themen (30\%), zu fachlichen Themen (29\%), zu pädagogischen Themen (12\%) oder zu Unterrichtsmethoden $(11 \%)$ besuchen und solche, die keine Fortbildungen $(18 \%)$ besuchen (Richter et al. 2013, S. 200). Thematische Interessen unterscheiden sich auch nach Schulform (Beck und Ullrich 1996): Lehrpersonen interessieren sich an Grundschulen z.B. eher für didaktische Themen als an Gymnasien, und Lehrpersonen an Grund-, Haupt- und Sonderschulen möchten besonders häufig in erzieherischen Fragen kompetenter werden.

\subsection{Fragestellung}

Der Forschungsstand (2.2) verweist auf ein Rahmenmodell, in dem verschiedene Merkmale der Fortbildungsangebote in einem komplexen Zusammenhang mit der Wirkung von Fortbildungen gesehen werden. In der Folge wurden Studien skizziert, welche die Nutzung von Fortbildungen in den Zusammenhang mit personenbezogenen, strukturellen oder inhaltlichen Merkmalen stellen. Diese Studien beziehen sich aber teils auf Gelegenheitsstichproben von Lehrpersonen, teils beschränken sie sich 
auf eine bestimmte Domäne (Schulfach). Die Daten werden bislang häufig durch die Befragung von Lehrpersonen gewonnen, die retrospektive Selbstauskünfte erteilen. Wie sich die Nutzung der Fortbildungen im komplexen Gesamtzusammenhang eines Lehrerinnen- und Lehrerbildungssystems (in Deutschland: Bundesland) darstellt, wurde bisher kaum erforscht. Eine signifikante Ausnahme bildet die Studie von Richter et al. (2020), die für Brandenburg einen Zusammenhang zwischen der absoluten Teilnehmendenzahl (als Indikator der Nutzung) und zeitlichen Merkmalen von Fortbildungen aufzeigt. Wie die Nutzung der Fortbildungen durch multiple strukturelle und inhaltliche Variablen (im Sinne von Merkmalen des Fortbildungsangebots) erklärt werden kann, wurde im deutschsprachigen Raum bislang nicht untersucht, obwohl angenommen wird, dass diese Angebotsmerkmale die Wahrnehmung und Nutzung der Fortbildungen beeinflussen. Dieses Desiderat wird aufgegriffen: Um ein aussagekräftiges Bild von möglichen Prädiktoren der Nachfrage an Fortbildungen zu zeichnen, werden mögliche Zusammenhänge unter schrittweiser Hinzunahme solcher Faktoren modelliert. Damit schließt die Studie an die Arbeit von Richter et al. (2020) an und erweitert diese um weitere strukturelle, erstmals aber auch inhaltliche Aspekte des Fortbildungsangebots als Prädiktoren dessen Nutzung.

Die absoluten Teilnehmendenzahlen (als Indikator der Nutzung) können nur begrenzt Auskunft geben: So bleibt offen, ob z. B. aus Gründen einer erreichten Kapazitätsobergrenze weniger Teilnehmende für ein Angebot zugelassen werden als es Interessierte hierfür gibt (d.h. die Nachfrage größer ist, als Teilnehmendenzahlen indizieren). Im Folgenden wird deshalb die tatsächliche Nachfrage (Zahl aller Anmeldungen) an einem Fortbildungsangebot betrachtet. Da diese Nachfrage hier als Reaktion auf das bestehende Angebot zu verstehen ist, scheint zudem relevant, die wechselseitige Bedingung von Angebot und Nutzung zu berücksichtigen. Um hierzu erste Hinweise zu erhalten, wird zusätzlich zur Nachfrage auch die Auslastung der Fortbildungen als Verhältnis aller angemeldeten Personen (Nachfrage) zu den tatsächlich verfügbaren Fortbildungsplätzen (Kapazität als ein Angebotsmerkmal) mit einbezogen. Die zentrale Forschungsfrage lautet demnach: Inwieweit lassen sich die Nachfrage und Auslastung von Fortbildungen in Baden-Württemberg durch strukturelle und/oder inhaltliche Merkmale des Angebots vorhersagen? Zur differenziellen Untersuchung von Nachfrage und Auslastung wird ein Datensatz mit schulexternen Fortbildungsangeboten herangezogen.

\section{Methode}

\subsection{Datengrundlage}

Grundlage für die Analysen bildet ein Datensatz, in dem für den Zeitraum eines kompletten Jahres (01.08.2016 bis 31.07.2017) Informationen zu $N=8953$ regionalen Fortbildungsangeboten in Verantwortung der Regierungspräsidien und Schulämter (Zuständigkeit: Kultusministerium) von Baden-Württemberg erfasst sind. Enthalten sind die Ausschreibungen der Fortbildungsangebote, auf die Teilnahmeinteressierte über einen zentralen Server zugreifen konnten, sowie Informationen zur maximalen Teilnehmendenzahl, zur Anzahl der zugelassenen Teilnehmenden sowie zur Zahl 
der Personen auf der Warteliste. Aus diesem Datensatz wurden diejenigen Fälle ausgewählt, die zur Beantwortung der Fragestellung (vgl. 2.3) dienlich sind. Da der Fokus dieses Beitrags auf strukturellen und inhaltlichen Merkmalen schulexterner Fortbildungen für Lehrerinnen und Lehrer liegt, wurden nur Angebote berücksichtigt, die sich erstens an Lehrpersonen und nicht an Inhabende von Funktionsstellen wie Schulleitende oder Schulpsychologinnen und -psychologen richteten. Zweitens wurden schulinterne Fortbildungen sowie Erlasslehrgänge und Abrufveranstaltungen nicht berücksichtigt, da für diese keine freie Anmeldung über das System möglich war. Zudem wurden fehlende Werte bereinigt (vgl. 3.3), sodass der Datensatz für die Analysen $N=6240$ Angebote umfasst.

\subsection{Beschreibung der Variablen}

Abhängige Variablen Entsprechend der in Kap. 2.3 formulierten Forschungsfrage wurde als erste abhängige Variable die Nachfrage als Anzahl aller Anmeldungen (Summe zugelassene Teilnehmende sowie Personen auf Warteliste) aus dem Datensatz gewonnen. Diese Variable entspricht der realen Nachfrage, weil sie die Anzahl aller Teilnahmeinteressierten repräsentiert, also all diejenigen Personen, die ein Angebot belegt hätten, wenn dessen Kapazität nicht begrenzt wäre. Als zweite abhängige Variable wurde die Auslastung als Quotient aus der Nachfrage und der Kapazität des Angebots (maximale Teilnehmendenzahl, die für die Fortbildung vorgesehen ist) gebildet. Diese Variable ist ein Indikator für die Passung von Nachfrage und Kapazität.

Unabhängige Variablen Zur Erklärung der Nachfrage und der Auslastung wurden als Prädiktoren jeweils die folgenden strukturellen und inhaltlichen Merkmale des Angebots berücksichtigt: Seitens der strukturellen Merkmale kann im Datensatz auf zeitliche Merkmale der Fortbildungen direkt oder nach Datentransformationen zugegriffen werden. Über Datum und Uhrzeiten sind der Wochentag sowie der Kalendermonat, an dem die Fortbildungen beginnen, zugänglich, und es kann eine Unterscheidung der eintägigen Veranstaltungen in halbtägige (bis zu vier Stunden) oder ganztägige (über vier Stunden) Fortbildungen getroffen werden. Fortbildungen, die drei oder mehr Tage dauern sowie Fortbildungsreihen, die sich in mehreren Teilen über einen längeren Zeitraum erstrecken, sind im Datensatz nicht zu unterscheiden und werden daher zusammengefasst. Schulformen, für welche die Fortbildungen geöffnet sind, werden in Grundschule, Hauptschule, Werkrealschule, Realschule, Gemeinschaftsschule, Gymnasium, Sonderpädagogisches Bildungs- und Beratungszentrum und Berufliche Schule unterschieden (Mehrfachzuordnungen möglich). Die Werkrealschule wird mit der strukturell ähnlichen Hauptschule zusammengefasst. Als inhaltliche Merkmale werden die Informationen zum Schulfach, auf das sich die Fortbildung bezieht, in fünf Gruppen an Fächern mit ähnlichem Profil zusammengefasst: MINT (z. B. Mathematik, Informatik, Naturwissenschaften, Technik), Sprachen (z.B. Deutsch, Englisch, Französisch, Latein), Geistes-/Sozialwissenschaften (z.B. Geschichte, Wirtschaft, Philosophie, Religionslehre), Ästhetik (z.B. Kunst, Musik, Sport) sowie fächerübergreifend (z.B. Allgemeine Didaktik). Zur Gewin- 
nung der Themen werden die inhaltlichen Angebotsbeschreibungen kriteriengeleitet nach inhaltlichen Kategorien kodiert (vgl. 3.3).

\subsection{Datenanalyse}

Die Auswertung der Daten erfolgt in zwei Schritten: Zunächst werden inhaltsanalytisch Fortbildungsthemen identifiziert, die anschließend als inhaltliche Merkmale in Regressionsanalysen zusammen mit strukturellen und weiteren inhaltlichen Merkmalen als Prädiktoren der Nachfrage und Auslastung herangezogen werden. Im Anschluss wird das statistische Auswertungsverfahren erläutert.

\subsubsection{Inhaltsanalyse der Fortbildungsthemen}

Als Ausgangspunkt wurden für eine zufällige Auswahl von Angeboten die in der Datenbank enthaltenen Beschreibungen zu Titel, Ziel und Programm der Fortbildungen untersucht. Mit dem Ziel, über thematische Kategorien den Inhalt der Fortbildung zu beschreiben, wurden auf Grundlage einer breit angelegten Exploration von zwei Kodierenden induktiv erste heuristische Analysekategorien im Modus der A-priori-Kategorienbildung (Kuckartz 2018) gewonnen. Über eine Konsensfindung wurden gemeinsam trennscharfe Kategorien festgelegt und jeweils unabhängig voneinander probeweise kodiert. Dabei wurde der Kodierleitfaden sukzessive angepasst, bis beide Kodierenden unabhängig voneinander zuverlässig gleiche Kategorien zugeordnet haben. Ein Angebot konnte potenziell mehreren Kategorien zugewiesen werden. Dabei wurde auf eine semantisch eindeutige Abgrenzung der Kategorien geachtet: So wurde z.B. eine Fortbildung mit dem Titel „Mündliche Prüfungen im Abitur gestalten“ den Kategorien ,Leistungsbeurteilung ' (Fokus: Ergebnis- und Prozessdiagnostik) und „Abschlussprüfung“ zugeordnet, das Angebot „Sonderpädagogische Diagnostik bei Kindern und Jugendlichen mit Auffälligkeiten im Bereich des Verhaltens“ hingegen der Kategorie ,Diagnostik“ (Fokus: Eigenschafts- und Verhaltensdiagnostik). Die Kategorie ,Qualifikation“ bezieht sich z. B. auf eine formale (Erst-)Qualifikation für Funktionsstellen oder für Beratungslehrpersonen.

Nach abschließender Definition und Beschreibung der Kategorien wurden zwei neue Kodierende in der Anwendung des Kodierleitfadens geschult, um auszuschließen, dass die Kodierungen durch implizite Absprachen beeinflusst werden (Krippendorff 2019). Von diesen unabhängigen Kodierenden wurde zur Prüfung der Beurteilerübereinstimmung eine Teilstichprobe von insgesamt 590 Fortbildungen $(9,4 \%)$ doppelt kodiert. Für die Berechnung der Beobachterübereinstimmung wird das auch bei systematischen Nicht-Übereinstimmungen robuste und spezifisch für Inhaltsanalysen entwickelte Maß Krippendorffs Alpha ( $\alpha$ ) (Hayes und Krippendorff 2007) verwendet. Für sieben Kategorien, die äußerst selten (jeweils weniger als 0,3\% der kodierten Angebote) kodiert wurden, liegt die Reliabilität unter dem von Krippendorff (2019) genannten Grenzwert von $\alpha=0,67$, weshalb sie aus den weiteren Analysen ausgeschlossen wurden. Die in den Analysen berücksichtigten Kategorien und die entsprechenden Reliabilitätswerte, die zufriedenstellend bis sehr gut sind und belastbare Analysen erlauben (Krippendorff 2019; Neuendorf 2002), können Tab. 1 entnommen werden. 
Tab. 1 Prädiktoren der Nachfrage sowie der Auslastung von Fortbildungsangeboten

Abhängige Variable

Nachfrage der Fortbildung

(Anzahl aller Anmeldungen)
Auslastung der Fortbildung

(Verhältnis von Nachfrage und Kapazität)

Prädiktoren $\quad$ Modell $1 \quad$ Modell $2 \quad$ Modell $3 \quad$ Modell $4 \quad$ Modell $1 \quad$ Modell $2 \quad$ Modell $3 \quad$ Modell 4

\section{Strukturelle Prädiktoren}

Dauer (Referenzkategorie: 0,5 Tag)

$\begin{array}{lllllllll}\text { 1 Tag } & \mathbf{3 , 1 1} & \mathbf{2 , 3 6} & \mathbf{3 , 0 3} & \mathbf{3 , 3 5} & \mathbf{0 , 1 4} & \mathbf{0 , 0 5} & 0,04 & \mathbf{0 , 0 5} \\ \text { 2 Tage } & \mathbf{4 , 4 1} & \mathbf{5 , 2 1} & \mathbf{5 , 8 7} & \mathbf{6 , 3 6} & \mathbf{0 , 3 0} & \mathbf{0 , 2 6} & \mathbf{0 , 2 5} & \mathbf{0 , 2 6} \\ \text { 3 oder } & \mathbf{1 , 2 4} & \mathbf{1 , 5 5} & \mathbf{1 , 7 0} & \mathbf{1 , 9 3} & \mathbf{0 , 2 1} & \mathbf{0 , 2 2} & \mathbf{0 , 2 1} & \mathbf{0 , 2 0}\end{array}$

mehr

Tage

Uhrzeit Beginn (Referenzkategorie: vormittags)

\begin{tabular}{|c|c|c|c|c|c|c|c|c|}
\hline \multicolumn{2}{|c|}{ Nachmittags $\mathbf{- 2 , 9 0}$} & $-3,34$ & $-2,88$ & $-2,59$ & $-\mathbf{0 , 1 0}$ & $-\mathbf{0 , 1 0}$ & $-\mathbf{0 , 1 0}$ & $-0,09$ \\
\hline \multicolumn{9}{|c|}{ Wochentag Beginn (Referenzkategorie: Montag) } \\
\hline Dienstag & $-0,31$ & $-0,32$ & $-0,38$ & $-0,29$ & $-0,05$ & $-0,04$ & $-0,04$ & $-0,04$ \\
\hline Mittwoch & $-0,32$ & $-0,27$ & $-0,40$ & $-0,50$ & $-0,09$ & $-0,07$ & $-0,07$ & $-\mathbf{0 , 0 7}$ \\
\hline Donnerstag & $-0,65$ & $-0,46$ & $-0,59$ & $-0,50$ & $-0,07$ & $-0,06$ & $-0,06$ & $-0,06$ \\
\hline Freitag & 0,13 & 0,33 & 0,16 & 0,17 & $-0,05$ & $-0,03$ & $-0,03$ & $-0,03$ \\
\hline Samstag & 14,19 & 14,87 & 14,49 & 14,24 & $-0,09$ & 0,01 & 0,01 & 0,03 \\
\hline Sonntag & $-9,65$ & $-8,31$ & $-7,65$ & $-6,94$ & $-0,46$ & $-0,39$ & $-0,38$ & $-0,36$ \\
\hline \multicolumn{9}{|c|}{ Monat Beginn (Referenzkategorie: Januar) } \\
\hline Februar & $-1,17$ & $-1,35$ & $-1,30$ & $-1,34$ & $-0,05$ & $-0,06$ & $-0,06$ & $-0,06$ \\
\hline März & 1,10 & 0,95 & 0,95 & 0,96 & $-0,02$ & $-0,03$ & $-0,03$ & $-0,02$ \\
\hline April & $-0,19$ & $-0,28$ & $-0,06$ & $-0,24$ & $-0,02$ & $-0,03$ & $-0,03$ & $-0,04$ \\
\hline Mai & $-0,52$ & $-0,36$ & $-0,20$ & $-0,16$ & $-0,01$ & 0,00 & 0,00 & $-0,00$ \\
\hline Juni & 0,35 & $-0,02$ & 0,29 & 0,26 & 0,01 & $-0,01$ & $-0,01$ & $-0,01$ \\
\hline Juli & 0,79 & 0,32 & 0,57 & 0,74 & 0,05 & 0,02 & 0,02 & 0,02 \\
\hline September & 5,77 & 5,36 & 5,46 & 5,35 & 0,06 & 0,04 & 0,05 & 0,04 \\
\hline Oktober & $-0,78$ & $-0,88$ & $-0,80$ & $-0,78$ & $-0,04$ & $-0,06$ & $-0,05$ & $-0,05$ \\
\hline November & $-0,05$ & $-0,21$ & $-0,22$ & $-0,07$ & $-0,01$ & $-0,03$ & $-0,02$ & $-0,02$ \\
\hline Dezember & 0,42 & 0,33 & 0,16 & 0,24 & $\mathbf{0 , 0 8}$ & 0,06 & 0,06 & 0,05 \\
\hline \multicolumn{9}{|c|}{ Schulform (Mehrfachzuordnungen möglich) } \\
\hline Grundschule & - & $-0,02$ & $-0,66$ & $-0,33$ & - & $-\mathbf{0 , 0 3}$ & $-\mathbf{0 , 0 3}$ & $-\mathbf{0 , 0 3}$ \\
\hline WHRRS & - & $-1,42$ & $-2,14$ & $-1,77$ & - & $-0,10$ & $-0,09$ & $-0,09$ \\
\hline Gymnasium & - & 2,34 & 2,13 & 2,13 & - & $\mathbf{0 , 1 4}$ & $\mathbf{0 , 1 5}$ & $\mathbf{0 , 1 5}$ \\
\hline $\begin{array}{l}\text { Gemein- } \\
\text { schaftsschule }\end{array}$ & - & $-0,80$ & $-0,41$ & $-0,53$ & - & $-\mathbf{0 , 0 3}$ & $-0,03$ & $-0,02$ \\
\hline $\begin{array}{l}\text { Berufliche } \\
\text { Schule }\end{array}$ & - & $-3,03$ & $-3,48$ & $-2,99$ & - & $-0,01$ & $-0,01$ & $-0,01$ \\
\hline Sonder- & - & $-0,14$ & $-0,81$ & $-0,54$ & - & 0,02 & 0,02 & 0,02 \\
\hline
\end{tabular}

schule

\section{Inhaltliche Prädiktoren}

Schulfach (Referenzkategorie: fächerübergreifende Themen)

\begin{tabular}{|c|c|c|c|c|c|c|c|}
\hline Ästhetik & - & - & $-1,70$ & $-1,87$ & - & - & 0,02 \\
\hline $\begin{array}{l}\text { Geistes-/ } \\
\text { Sozialwis- } \\
\text { senschaft }\end{array}$ & - & - & $-2,15$ & $-2,40$ & - & - & 0,00 \\
\hline
\end{tabular}


Tab. 1 (Fortsetzung)

\begin{tabular}{|c|c|c|c|c|c|c|c|c|}
\hline \multirow[b]{3}{*}{ Prädiktoren } & \multicolumn{8}{|c|}{ Abhängige Variable } \\
\hline & \multicolumn{4}{|c|}{$\begin{array}{l}\text { Nachfrage der Fortbildung } \\
\text { (Anzahl aller Anmeldungen) }\end{array}$} & \multicolumn{4}{|c|}{$\begin{array}{l}\text { Auslastung der Fortbildung } \\
\text { (Verhältnis von Nachfrage und Kapazität) }\end{array}$} \\
\hline & Modell 1 & Modell 2 & Modell 3 & Modell 4 & Modell 1 & Modell 2 & Modell 3 & Modell 4 \\
\hline MINT & - & - & $-3,46$ & $-3,45$ & - & - & 0,06 & 0,06 \\
\hline Sprachen & - & - & $-3,03$ & $-3,56$ & - & - & $-0,02$ & $-0,01$ \\
\hline \multicolumn{9}{|c|}{ Thema (Mehrfachzuordnungen möglich) } \\
\hline $\begin{array}{l}\text { Abschluss- } \\
\text { prüfung } \\
(0,83)\end{array}$ & - & - & - & 2,00 & - & - & - & $-0,00$ \\
\hline $\begin{array}{l}\text { Austausch- } \\
\text { treffen } \\
(0,78)\end{array}$ & - & - & - & 0,58 & - & - & - & $-0,03$ \\
\hline $\begin{array}{l}\text { Supervision } \\
(0,83)\end{array}$ & - & - & - & $-3,58$ & - & - & - & $-0,09$ \\
\hline $\begin{array}{l}\text { Curriculum } \\
(0,89)\end{array}$ & - & - & - & $\mathbf{1 , 0 8}$ & - & - & - & $-0,02$ \\
\hline $\begin{array}{l}\text { Diagnostik } \\
(0,71)\end{array}$ & - & - & - & 2,22 & - & - & - & 0,06 \\
\hline $\begin{array}{l}\text { Didaktik } \\
(0,67)\end{array}$ & - & - & - & $-1,18$ & - & - & - & $-0,04$ \\
\hline $\begin{array}{l}\text { Gesundheit } \\
(0,91)\end{array}$ & - & - & - & 3,42 & - & - & - & 0,06 \\
\hline $\begin{array}{l}\text { Inklusion } \\
(0,89)\end{array}$ & - & - & - & $-2,14$ & - & - & - & $-0,05$ \\
\hline $\begin{array}{l}\text { Leistungs- } \\
\text { beurteilung } \\
(0,82)\end{array}$ & - & - & - & 2,92 & - & - & - & 0,03 \\
\hline $\begin{array}{l}\text { Digitali- } \\
\text { sierung } \\
(0,69)\end{array}$ & - & - & - & $-2,83$ & - & - & - & $-0,04$ \\
\hline $\begin{array}{l}\text { Prävention } \\
(0,70)\end{array}$ & - & - & - & $-0,92$ & - & - & - & $-0,07$ \\
\hline $\begin{array}{l}\text { Qualifi- } \\
\text { kation } \\
(0,72)\end{array}$ & - & - & - & 4,27 & - & - & - & 0,32 \\
\hline $\begin{array}{l}\text { Recht/ } \\
\text { Gesetz } \\
(0,96)\end{array}$ & - & - & - & 4,06 & - & - & - & 0,17 \\
\hline $\begin{array}{l}\text { Hetero- } \\
\text { genität } \\
(0,68)\end{array}$ & - & - & - & $-0,05$ & - & - & - & $-0,04$ \\
\hline$R^{2}$ & 0,061 & 0,077 & 0,086 & 0,103 & 0,120 & 0,155 & 0,159 & 0,186 \\
\hline $\begin{array}{l}\text { Korrigiertes } \\
R^{2}\end{array}$ & 0,058 & 0,074 & 0,081 & 0,097 & 0,117 & 0,151 & 0,154 & 0,179 \\
\hline F-Statistik & $\begin{array}{l}20.169 \\
(20 ; 6204)\end{array}$ & $\begin{array}{l}20.018 \\
(26 ; 6198)\end{array}$ & $\begin{array}{l}19.261 \\
(30 ; 6155)\end{array}$ & $\begin{array}{l}16.061 \\
(44 ; 6141)\end{array}$ & $\begin{array}{l}38.682 \\
(20 ; 5682)\end{array}$ & $\begin{array}{l}39.918 \\
(26 ; 5676)\end{array}$ & $\begin{array}{l}35.488 \\
(30 ; 5640)\end{array}$ & $\begin{array}{l}29.135 \\
(44 ; 5626)\end{array}$ \\
\hline
\end{tabular}

Berichtet werden die unstandardisierten Regressionskoeffizienten $(\beta)$; fettgedruckt $=p<0,05 ; R^{2}=$ auf-

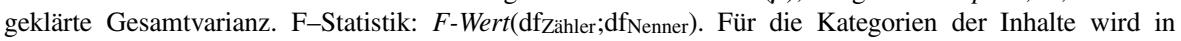
Klammern jeweils die Beurteilerübereinstimmung (Krippendorff's Alpha ( $\alpha$ ), vgl. Kap. 3.3) berichtet 


\subsubsection{Datenaufbereitung und statistische Verfahren}

In der Mehrheit lagen die Informationen vollständig vor oder wurden im Rahmen der Inhaltsanalyse (s. oben) kodiert. Fehlende Werte treten im unbereinigten Gesamtdatensatz mit $N=8953$ nur in den Angaben zur maximalen Teilnehmendenzahl auf ( $n=529$; entspricht 8,5\% Ausfall auf Ebene der Fortbildungen), die vermutlich durch unsystematische Versäumnisse beim Verwalten der Datenbank entstanden sind. Da diese Informationen grundlegend für die Berechnung der abhängigen Variable sind, konnten die entsprechenden Fortbildungen in der Auswertung nicht weiter berücksichtigt werden. Von fall- oder paarweisem Ausschluss wird im Umgang mit fehlenden Werten in der Regel abgeraten (Lüdtke et al. 2007). Jedoch muss die interne Validität einer Studie nicht per se durch fehlende Werte beeinträchtigt werden, sofern die praktische Bedeutung der fehlenden Werte unabhängig von den modellierten Parametern ist (Graham 2009). Daher wurde mittels $\chi^{2}$-Test geprüft, ob sich Fortbildungen mit fehlenden Werten in der maximalen Teilnehmendenzahl statistisch signifikant von anderen Fortbildungen unterscheiden. Da bei umfangreicheren Stichproben kleine Unterschiede eher signifikant ausfallen, wurde zusätzlich die Effektstärke der Unterschiede über Cohen's $\omega$ berechnet (Eid et al. 2015). Es ergeben sich meist signifikante Unterschiede, die mit einer Effektstärke $\omega \leq 0,14$ aber durchweg als klein (Cohen 1988) und damit als vernachlässigbar einzustufen sind, weshalb Fälle mit fehlender maximaler Teilnehmendenzahl ausgeschlossen wurden.

Anschließend wurden die Modellvoraussetzungen für die Regressionsanalysen überprüft (Eid et al. 2015). Die Prüfung auf Multikollinearität zeigt hohe Korrelationen zwischen den Schulformen Realschule und Werkreal- bzw. Hauptschule $(r=0,922)$. Diese wurden zur Vermeidung von Suppressionseffekten (Schneider 2009) zusammengefasst, was angesichts des Schulsystems in Baden-Württemberg auch inhaltlich plausibel erscheint.

Zur Berechnung gemeinsamer und spezifischer Effekte der Prädiktoren auf die beiden abhängigen Variablen (Nachfrage und Auslastung) wurden jeweils vier lineare Regressionsmodelle erstellt. Es wurden schrittweise zunächst strukturelle Variablen (zeitliche Merkmale in einem ersten Regressionsmodell, dann der Schulformbezug in einem zweiten Modell) und daraufhin inhaltliche Variablen (zunächst der Fachbezug in einem dritten Modell und dann die Themen der Fortbildungen in einem vierten Modell) auf ihr erklärendes Potenzial für die beiden abhängigen Variablen untersucht. Die Abfolge der sequenziellen Hinzunahme an Prädiktoren folgt dem Anspruch, zunächst die von Richter et al. (2020) aufgezeigte prädiktive Bedeutung zeitlicher Merkmale zu replizieren und sodann unter Kontrolle des weiteren Strukturmerkmals der Schulform erstmals auch inhaltliche Merkmale (Schulfach und Thema) auf ihr zusätzliches erklärendes Potenzial hin zu untersuchen. Alle Analysen wurden mit der Open Source Software R (R Core Team 2019) durchgeführt, die Regressionsanalysen dabei mit dem R-Paket lavaan (Rosseel 2012). 


\section{Ergebnisse}

Das Forschungsanliegen der Arbeit ist, die Nachfrage an den Fortbildungsangeboten sowie die Auslastung durch strukturelle wie inhaltliche Merkmale zu erklären. Es werden dafür zunächst diejenigen deskriptiven Befunde skizziert, die für das Verständnis der nachfolgenden Regressionsanalysen erforderlich sind.

\subsection{Deskriptive Analysen}

Deskriptive Analysen der zeitlichen Merkmale der Fortbildungen zeigen, dass die meisten der 6240 Angebote (46,5\%) einen halben Tag dauern, etwas über ein Drittel der Veranstaltungen ist eintägig. Die zweitägigen Fortbildungen machen lediglich 2,8\% der Angebote aus. Die Hälfte der Angebote beginnt am Vormittag (50,2\%). Die meisten Fortbildungen finden in der Mitte der Woche statt (Dienstag: 20,5\%, Mittwoch: 37,2\%, Donnerstag: 20,4\%); zu Beginn bzw. am Ende der Woche sind es weniger Angebote (Montag: 14,6\%, Freitag: 7,0\%, Samstag: 0,3\%, Sonntag: $0,1 \%)$. Die Fortbildungen finden überwiegend außerhalb der unterrichtsfreien Zeit (Ferien) statt. Dies spiegelt sich auch in der Verteilung auf das Schuljahr wider: Die meisten Angebote beginnen am Anfang des Schuljahres im Oktober (15,8\%) und November $(17,0 \%)$ sowie im Sommerhalbjahr in den Monaten ohne Schulferien. Keine Veranstaltungen finden im Monat August statt, in dem die Sommerferien lagen; ebenfalls wenige im September $(3,4 \%)$, in dem teils noch Sommerferien waren; im Dezember beginnen 6,5\% der Angebote.

Der Großteil der Fortbildungen ist für Lehrpersonen mehrerer Schulformen geöffnet. Weit über die Hälfte der Fortbildungsangebote $(60,6 \%)$ ist für Gemeinschaftsschulen geöffnet. Etwas weniger Angebote sind für Werkrealschule, Hauptschule und Realschule (41,4\%), für die Grundschule (41,0\%), für das Gymnasium $(35,0 \%)$ oder für Sonderpädagogische Bildungs- und Beratungszentren (Sonderschulen) $(34,8 \%)$ geöffnet, und für Berufliche Schulen sind es etwas weniger als ein Viertel der Angebote $(22,1 \%)$.

Die Analysen zum Fachbezug zeigen, dass die meisten der Fortbildungen (35,1\%) fächerübergreifend sind oder keinen konkreten Fachbezug haben. Von den fachspezifischen Veranstaltungen werden die meisten im Bereich Mathematik, Informatik, Naturwissenschaft oder Technik (MINT) angeboten (22,4\%). Im Bereich Sprachen $(17,0 \%)$, Ästhetik $(13,1 \%)$ und in Geistes- oder sozialwissenschaftlichen Fächern $(12,4 \%)$ gibt es weniger Angebote.

Die Mehrzahl der Angebote finden zu den Themen Didaktik/Methodik (44,5\%) sowie Curriculum (24,9\%) statt. Während Angebote zum Umgang mit Heterogenität noch recht häufig sind $(13,6 \%)$, werden bildungspolitisch stark forcierte Themen wie Inklusion $(4,7 \%)$ oder Digitalisierung $(8,9 \%)$ eher selten ausgebracht. Zum Thema Prävention (z. B. im Umgang mit herausfordernden Schülerinnen und Schülern) werden 3,6\% der Angebote gemacht. Dem Thema Recht und Gesetz $(0,1 \%)$ sind nur wenige Angebote gewidmet. Zur Qualifikation für Funktionsstellen und für besondere Aufgaben werden 3,0\% der Fortbildungen angeboten.

Die Analysen zur abhängigen Variable der Nachfrage zeigen, dass es durchschnittlich pro Fortbildung im arithmetischen Mittel $M=10,64$, im Median $M d n=8$ 
$(S D=13,36 ; \min =0 ; \max =252)$ angemeldete Personen gibt; davon stehen durchschnittlich $M=1,04(M d n=0 ; S D=3,49 ; \min =0 ; \max =95)$ auf einer Warteliste (Fortbildung überbucht). Die Kapazität (Anzahl der Teilnahmeplätze) liegt im Mittel bei $M=23,74(M d n=20 ; S D=17,43 ; \min =1 ; \max =300)$. Durchschnittlich liegt die Auslastung der Fortbildungen bei $M=0,44(M d n=0,38 ; S D=0,41 ; \min =0$; $\max =6,36$ ). Das heißt, im Durchschnitt kommt auf zwei Teilnahmeplätze nur etwa eine interessierte Person; im Schnitt gibt es also doppelt so viele Plätze wie nachgefragt werden.

\subsection{Prädiktoren der Nachfrage sowie der Auslastung}

In Modell 1 (Tab. 1) werden zeitliche Merkmale der Fortbildung (Dauer sowie Uhrzeit, Wochentag und Monat des Beginns) als Prädiktoren angenommen. Im Ergebnis zeigt die Dauer der Fortbildung einen signifikanten positiven Effekt sowohl auf die Nachfrage sowie auf die Auslastung: Veranstaltungen mit kürzerer Dauer (bis zu 4h) haben eine geringere Nachfrage sowie Auslastung als ganztägige Fortbildungen. Auch Veranstaltungen, die 2 oder 3 oder mehr Tage dauern, sind stärker ausgelastet und werden stärker nachgefragt. Veranstaltungen mit einer Uhrzeit des Beginns am Vormittag werden besonders häufig nachgefragt, ein Beginn am Nachmittag verweist im Vergleich auf geringere Nachfrage. Es zeigen sich für zwei Wochentage des Beginns statistisch signifikante Effekte: Angebote mit Beginn am Montag, an dem es vergleichsweise weniger Kapazität gibt (vgl. 4.1), haben eine besonders hohe Auslastung. Mit Beginn am Samstag ist die Nachfrage vergleichsweise sehr hoch, die Auslastung aber nicht höher. Insbesondere zeigt sich eine geringe Auslastung an den Wochenenden, auch wenn hier angesichts geringer Fallzahlen kaum belastbare Befunde vorliegen. Der Monat, in dem das Fortbildungsangebot jeweils beginnt, kann kaum zur Erklärung der abhängigen Variablen beitragen. Ein Beginn im September weist auf eine höhere Nachfrage hin, ein Beginn im Dezember auf eine im Vergleich etwas höhere Auslastung. Insgesamt können über zeitliche Merkmale 5,8\% der Gesamtvarianz der Nachfrage sowie 11,7\% der Gesamtvarianz in der Auslastung erklärt werden.

In Modell 2 zeigt sich weiterhin, dass auch die Schulform einen statistisch signifikanten Beitrag zur Erklärung der abhängigen Variablen leistet. Bei den Veranstaltungen, die (auch) für die Schulformen Werkreal-, Haupt- oder Realschule ausgeschrieben sind, sind Nachfrage sowie Auslastung geringer; bei Fortbildungen, die für das Gymnasium geöffnet sind, hingegen höher. Für die Beruflichen Schulen fällt auf, dass die Nachfrage vergleichsweise gering ist, die Auslastung aber nicht: Die mittlere Anzahl der Teilnahmeplätze ist für berufliche Schulen niedriger. Unter Berücksichtigung der Schulform fällt der Einfluss der zeitlichen Merkmale Dauer und Wochentag auf die Auslastung etwas geringer aus als bei deren isolierter Betrachtung (Modell 1 Auslastung). Für die Nachfrage zeigt sich kein einheitliches Bild. Die aufgeklärte Gesamtvarianz steigt in Modell 2 auf 7,4\% (Nachfrage) bzw. $15,1 \%$ (Auslastung), die Schulform hat also über die zeitlichen Merkmale hinaus erklärendes Potenzial.

Zu den in Modell 3 eingeführten Fächern zeigt sich, dass Fortbildungen zu fächerübergreifenden Themen stärker nachgefragt werden als solche zu speziellen Fächern. 
Bezüglich der Auslastung haben allerdings nur die MINT-Fächer (Mathematik, Informatik, Naturwissenschaften, Technik) einen statistisch signifikanten zusätzlichen Erklärungswert für Unterschiede zwischen den Fortbildungsangeboten, und dieser ist zudem gering. Die aufgeklärte Gesamtvarianz steigt für die Nachfrage auf 8,1\%, für die Auslastung unwesentlich auf 15,4\%.

Bezüglich der in Modell 4 zusätzlich eingeführten Themen der Fortbildungen zeigen sich einige statistisch signifikante Effekte. Während allerdings die Themen Abschlussprüfung, Gesundheit und Leistungsbeurteilung jeweils eine höhere Nachfrage prädizieren, werden entsprechende Fortbildungen im Verhältnis zu den Teilnahmeplätzen nicht stärker ausgelastet. Fortbildungen zur Qualifikation (z. B. Beratungslehrpersonenausbildung) werden häufig nachgefragt und sind am stärksten ausgelastet. Auch bezüglich der Angebote zum Thema Recht und Gesetz zeigt sich eine höhere Nachfrage und Auslastung. Eher gering ist diese bei Angeboten zu Themen wie z.B. sonderpädagogische Förderung und Inklusion sowie Digitalisierung. Auch für den am häufigsten angebotenen Themenkomplex der Methodik und Didaktik werden mehr Teilnahmeplätze angeboten als nachgefragt. Am niedrigsten ist die Auslastung beim Thema Supervision, bei dem auch die Nachfrage gering ist. Durch die Aufnahme der Themen in das Regressionsmodell ist der kleine Effekt eines Beginns im Monat Dezember für die Auslastung nicht länger signifikant - andere Prädiktoren behalten ihr Erklärungspotenzial. Unter Hinzunahme der Themen steigt die aufgeklärte Gesamtvarianz der Nachfrage auf 9,7\%, bzw. für die Auslastung auf 17,9\%; inhaltliche Merkmale tragen also mit zur Aufklärung der Varianz in den abhängigen Variablen bei.

\section{Zusammenfassung und Diskussion}

Ziel der Studie war, Nachfrage und Auslastung von Fortbildungen durch strukturelle und inhaltliche Angebotsmerkmale zu prognostizieren. Damit sollte erstmals über rein zeitliche Angebotsmerkmale hinaus untersucht werden, welche weiteren Merkmale die Nachfrage an Fortbildungen und deren Auslastung erklären können.

Zusammenfassend erfahren Veranstaltungen mit längerer Dauer von einem ganzen Tag oder auch mehreren Tagen eine höhere Nachfrage sowie Auslastung als die überwiegend angebotenen halbtägigen Fortbildungen. Dies korrespondiert mit Befunden eines positiven Zusammenhangs von Angebotsdauer und Teilnehmendenzahl (Richter et al. 2020). Da kurzzeitige, sogenannte, one-shot'-Fortbildungen kaum als nachhaltig gelten (Lipowsky 2014) und Lehrpersonen insbesondere längere Fortbildungen nachfragen (vgl. 3; Richter et al. 2020), stellt sich die Frage, ob und wie Letztere strukturell möglich gemacht werden könnten und ob diese bildungspolitisch (überhaupt) gewünscht sind. Während Richter et al. (2020) den Fokus auf Fortbildungen zum Thema Unterricht legten, erklärt sich vorliegend unter Einbezug der vollen thematischen Breite an Fortbildungen eine höhere Nachfrage sowie Auslastung längerer Fortbildungen auch durch die adressierten Themen - etwa wenn Fortbildungen zur Qualifikation für besondere Aufgaben (z. B. Funktionsstellen) i.d. R. länger sind. Der Effekt der Dauer bleibt allerdings auch unter Kontrolle der Themen bestehen. 
Die deskriptiven Analysen zur Uhrzeit des Beginns der Veranstaltungen zeichnen ein bundeslandspezifisches Bild: Während in anderen Lehrerbildungssystemen Fortbildungen offenbar eher in der Mittagszeit sowie am frühen Nachmittag angeboten werden (Amrhein und Badstieber 2014; Daschner und Hanisch 2019; Richter et al. 2020) und somit eher außerhalb der Unterrichtszeit stattfinden, beginnen diese in Baden-Württemberg nahezu in gleichem Umfang am Vor- und Nachmittag. Angebote mit Beginn am Vormittag sind besonders stark nachgefragt. Dieser Befund deckt sich mit Ergebnissen von Richter et al. (2020) und mit Studien aus der allgemeinen Weiterbildungsforschung (Schmidt-Lauff 2008, 2018). Zu kurz greifen würde allerdings, Fortbildungen nur vormittags beginnen zu lassen: Neben weiteren Angebotsmerkmalen haben etwa auch die Teilnahmemotivation oder gesetzliche Regelungen (z. B. Arbeitszeitmodell) einen relevanten Einfluss auf die Teilnahme.

Zum Wochentag des Fortbildungsbeginns zeigt sich: Fortbildungen mit Beginn an Samstagen haben eine besonders hohe Nachfrage. Die Auslastung ist allerdings nicht besonders hoch. Ein Grund könnte sein, dass an Samstagen eher spezifische Formate wie Fachtagungen mit vielen Teilnehmenden, aber auch hoher Kapazität, angeboten werden. Mit Beginn am Montag, an dem vergleichsweise wenige Angebote stattfinden, ist die Auslastung besonders hoch, mit Beginn am Mittwoch, mit vergleichsweise großem Angebot, hingegen gering. Da es in anderen Bundesländern zentrale Vorgaben zu für Fortbildungen vorgesehenen Wochentagen gibt (z. B. für Brandenburg: Richter et al. 2020), ist ein Vergleich kaum möglich. Das Ergebnis verweist allerdings auf die potenzielle Relevanz, die Wochenstruktur in der Fortbildungsplanung zu berücksichtigen. Der Monat, in dem eine Fortbildung beginnt, trägt nur marginal zur Aufklärung der abhängigen Variablen bei. Die Ergebnisse entsprechen tendenziell aber dem Befund von Richter et al. (2020), wonach die Teilnehmendenzahl zu Beginn und zum Ende des Schuljahres höher ist.

In Bezug auf die Schulform zeigt sich für Angebote für Grundschulen, Gemeinschaftsschulen oder Haupt- und Werkrealschulen eine geringere, bezogen auf die Gymnasien hingegen eine höhere Auslastung. Für berufliche Schulen ließe sich auf Grundlage der geringeren Nachfrage vermuten, dass diese Angebote weniger gefragt sind - es zeigt sich aber eine durchschnittliche Auslastung der Angebote, sodass der Grund für die niedrigeren Teilnehmendenzahlen auch in der geringeren Kapazität der Angebote liegen können. In anderen Studien zeigen sich hingegen keine signifikanten Schulformunterschiede hinsichtlich der Teilnahme (Richter 2011). Da die Fortbildungen häufig für Lehrpersonen mehrerer Schulformen geöffnet sind und im Datensatz Mehrfachzuordnungen vorliegen, sind diese Ergebnisse unter Vorbehalt zu interpretieren. Weiterführende Aussagen würden erfordern, über die im Datensatz enthaltenen Angaben hinaus auch personengebundene Merkmale der Teilnehmenden - wie z.B. die Schulform, an der sie unterrichten - einzubeziehen.

Analysen zum Fachbezug zeigen, dass Veranstaltungen im Bereich MINT im Vergleich zu fächerübergreifenden Fortbildungen etwas stärker ausgelastet sind. Der Effekt ist zwar gering, entspricht aber den wenigen Befunden zur Nutzung von Fortbildungen nach Fachbezug, wonach Lehrpersonen häufiger oder bevorzugt Fortbildungen mit konkretem Fachbezug besuchen (Jäger und Bodensohn 2007; Ministerium für Kultus, Jugend und Sport Baden-Württemberg 2017). Dies spiegelt sich nicht im Angebot wider: Etwa die Hälfte der Fortbildungen weist keinen explizi- 
ten Fachbezug auf (Johannmeyer et al. 2019b), weshalb eine Sensibilität für den Fachbezug bei der Angebotsplanung nötig erscheint.

Bezüglich der Themen erstaunt, dass Fortbildungen zu derzeit bildungspolitisch stark forcierten Bereichen wie Inklusion bzw. sonderpädagogische Förderung oder Digitalisierung vergleichsweise selten angeboten werden und zudem gering ausgelastet sind. Damit werden die administrativen Ziele offenbar doppelt unterlaufen: Die gesetzte Relevanz der Themen spiegelt sich nicht in der Quantität des einschlägigen Angebots nieder und die wenigen Angebote werden schlecht angenommen. Dieser Befund steht im Kontrast zu Studien, nach welchen Angebote zu diesen Themen international verstärkt nachgefragt werden (Gargarina und von Saldern 2010; OECD 2009). Hierin zeigt sich offenbar die hochgradige Kontextspezifität im Verhältnis von Angebot und Nutzung in spezifischen Lehrerbildungssystemen. Auch gibt es offenbar eine merkliche Diskrepanz zwischen dem intendierten und implementierten Curriculum in der Lehrerinnen- und Lehrerbildung (Cramer et al. 2020).

Vorliegend erweisen sich Angebote für besondere Aufgaben (z.B. Qualifikation für Funktionsstellen oder für Beratungslehrpersonen) als besonders ausgelastet. Dies könnte auch auf die Notwendigkeit des Besuchs von formal erforderlichen Fortbildungen für die Aufnahme der Tätigkeit einer Beratungslehrperson zurückzuführen sein. Während in anderen Studien bislang der Fokus häufig auf Fortbildungen zum Thema Unterricht gelegt wurde, wurden vorliegend auch Fortbildungen zu anderen Themen untersucht. Wenn bildungspolitisch Akzente im Fortbildungsangebot gesetzt werden sollen, so sind Überlegungen anzustellen, wie entsprechende Angebote ausgelastet werden können. Wenn, wie hier gezeigt, die Auslastung der Angebote insgesamt sehr gering ist und nur etwa eine teilnehmende Person auf zwei Teilnahmeplätze kommt, geraten die Modalitäten der Angebotsplanung in den Blick, aber es stellt sich auch die Frage nach Gründen, die eine Teilnahme erschweren oder verhindern (vgl. Richter et al. 2018).

Nicht nur Merkmale des Angebots sind relevante Prädiktoren der Auslastung. Insbesondere deuten Varianzaufklärungen von maximal 17,9\% in den abhängigen Variablen darauf hin, dass weitere bedeutsame Aspekte die Fortbildungsteilnahme beeinflussen. Inhaltlich könnten diese beispielsweise eine Präferenz der Teilnehmenden für methodisch-didaktische Ausrichtungen wie z. B. die Arbeit in Kleingruppen sein, strukturell etwa die Nähe der Fortbildung zum Wohn- oder Arbeitsort. Auch könnte eine Analyse von Interaktionseffekten der betrachteten Merkmale in weiterführender Forschung ertragreich sein. Weiterhin werden Ursachen für die Fortbildungsteilnahme auch außerhalb des Angebots liegen. So ist die Entscheidung für eine Teilnahme bspw. auch durch motivationale Aspekte bedingt: Berufsbezogene Aspekte spielen eine zentrale Rolle (Richter et al. 2019), aber z.B. auch der Wunsch nach sozialen Kontakten (Kao et al. 2011; Johannmeyer et al. 2019c) sowie äußere Faktoren (z.B. Hindernis aufgrund zu geringer Unterrichtsversorgung; Richter et al. 2018). Offen ist zudem, inwiefern Aspekte wie eine Präferenz für bestimmte Dozierende die Teilnahmeentscheidung beeinflussen. Als eine mögliche Erklärung der Teilnahme wurden von Richter et al. auch Erwartungs-Wert-Ansätze (Eccles 2005) herangezogen, welchen zufolge im komplexen Gefüge von Gründen der Entscheidung auch der antizipierte Wert der Teilnahme an einer Fortbildung (z.B. utilitaristischer Wert, persönlicher Wert) eine Rolle spielt. 
Hierin zeigt sich die zu Beginn antizipierte Relevanz, nicht nur die absoluten Teilnehmendenzahlen zu betrachten, sondern auch die Nachfrage sowie die reale Auslastung. Nur dann gerät auch eine mögliche wechselseitige Bedingung von Angebot und Nachfrage in den Blick. In Interviews mit Verantwortlichen für die Fortbildungsplanung in Baden-Württemberg zeigt sich etwa, dass bereits im Planungshandeln der Bedarf und damit die potenzielle Nachfrage zumindest teilweise berücksichtigt wird (Johannmeyer et al. 2019a). Eine höhere Auslastung könnte daher auch durch die Angebotsplanung im Voraus bedingt sein und darf nicht pauschal als Indikator für die tatsächliche Nachfrage fehlinterpretiert werden.

\section{Fazit und Ausblick}

Insgesamt liegt mit dieser Studie erstmals für ein Lehrerbildungssystem in Deutschland eine hinsichtlich zeitlicher und inhaltlicher Angebotsmerkmale umfassende Analyse des Fortbildungsangebots eines Bundeslandes für ein gesamtes Jahr vor. Es konnten Zusammenhänge zwischen strukturellen sowie inhaltlichen Merkmalen der Fortbildungen mit der Nachfrage sowie mit der Auslastung aufgezeigt werden. An der Bedarfserhebung und Planung beteiligte Akteure können für mögliche Ursachen der Auslastung sensibilisiert werden und bei einer geringen Passung von Angebot und Nachfrage das vorgehaltene Angebot auf dieser Grundlage kritisch diskutieren.

Mit der Programmanalyse liegt eine ökonomische und verlässliche Methode vor, da die Daten leicht zugänglich sind, der Koordinationsbedarf mit Akteuren im System gering ist und Analysen auch rückwirkend möglich sind (Schrader und Ioannidou 2011, S. 259f.). Die Methode vermag es jedoch nicht, Einblick in die „Inhalte, die Motive und die Formen des Handelns von Akteuren“ zu geben (Schrader 2011, S. 150). Über die vorliegend berücksichtigten strukturellen und thematischen Prädiktoren der Nachfrage und Auslastung hinaus sind daher auch personengebundene (z. B. motivationale) und auf die (rechtlichen) Rahmenbedingungen bezogene Aspekte zur Erklärung der Nachfrage heranzuziehen. Der hier gewählte Fokus sensibilisiert dafür, dass verschiedene Merkmale des Angebots mit über dessen Nachfrage bestimmen können.

Vorliegend wurden regionale Fortbildungen für Lehrpersonen in Verantwortung der Schulämter und Regierungspräsidien berücksichtigt. In weiteren Analysen sollte zukünftig auch der Bereich der berufsbegleitenden Fortbildung von Schulleitungen thematisiert werden. Weitere Einblicke in das komplexe Gesamtsystem könnten durch Einbezug zentraler Fortbildungen oder von Angeboten freier Träger gewonnen werden. Aufgrund der spezifischen Fortbildungssysteme können die Befunde nur bedingt auf andere Bundesländer übertragen werden (Nolda 2018, S. 445). Geboten erscheint, länderübergreifend in der Forschung zu kooperieren, um mehr als kumulativen Erkenntnisgewinn zu erreichen.

Noch detailliertere Einblicke in die Strukturen von Angebot und Nutzung könnten gewonnen werden, wenn künftig (seitens der Bildungsadministration) zusätzliche Informationen zu den Teilnehmenden (z. B. zum Nutzungsverhalten, zur Teilnahmemotivation, zu bisherigen Besuchen von Fortbildungen) erfasst würden: Nachdem sich das Angebot nach Regionen stark unterscheidet und die Teilnehmenden teils 
große Anfahrtswege auf sich nehmen (Cramer et al. 2019), könnten Nachfrage und Auslastung im Zusammenhang mit Informationen über die Teilnehmenden u. a. auch mit durch geografische Aspekte prädiziert werden. Wenn Analysen des Angebots nicht wie bisher nur einmalig (vgl. z. B. Daschner und Hanisch 2019; Johannmeyer et al. 2019b; Richter et al. 2020), sondern im Längsschnitt durchgeführt würden, könnte ein solches Monitoring der Angebotsstrukturen wertvolle Steuerungsinformationen für das Gesamtsystem bereithalten, um etwa die Passung von Angebot und Nachfrage zu erhöhen. Dies erscheint angesichts der großen Bedeutung von Fortbildungen für den Aufbau und den Erhalt berufslebenslanger Professionalität (Cramer 2019) von hoher Relevanz.

Funding Open Access funding enabled and organized by Projekt DEAL.

Open Access Dieser Artikel wird unter der Creative Commons Namensnennung 4.0 International Lizenz veröffentlicht, welche die Nutzung, Vervielfältigung, Bearbeitung, Verbreitung und Wiedergabe in jeglichem Medium und Format erlaubt, sofern Sie den/die ursprünglichen Autor(en) und die Quelle ordnungsgemäß nennen, einen Link zur Creative Commons Lizenz beifügen und angeben, ob Änderungen vorgenommen wurden.

Die in diesem Artikel enthaltenen Bilder und sonstiges Drittmaterial unterliegen ebenfalls der genannten Creative Commons Lizenz, sofern sich aus der Abbildungslegende nichts anderes ergibt. Sofern das betreffende Material nicht unter der genannten Creative Commons Lizenz steht und die betreffende Handlung nicht nach gesetzlichen Vorschriften erlaubt ist, ist für die oben aufgeführten Weiterverwendungen des Materials die Einwilligung des jeweiligen Rechteinhabers einzuholen.

Weitere Details zur Lizenz entnehmen Sie bitte der Lizenzinformation auf http://creativecommons.org/ licenses/by/4.0/deed.de.

\section{Literatur}

Amrhein, B., \& Badstieber, B. (2014). Lehrerfortbildungen zur Inklusion - eine Trendanalyse. Gütersloh: Bertelsmann.

Beck, C., \& Ullrich, H. (1996). Fortbildungsinteressen von Lehrenden. Die Deutsche Schule, 88(2), 198-213.

Boeren, E., Nicaise, I., \& Baert, H. (2010). Theoretical models of participation in adult education: the need for an integrated model. International Journal of Lifelong Education, 29(1), 45-61.

Böttcher, W., Heinemann, U., Kubina, C., \& Priebe, B. (Hrsg.). (2019). Steuerung und Qualitätsentwicklung im Fortbildungssystem. Hannover: Klett.

Cohen, J. (1988). Statistical power analysis for the behavioral sciences (2. Aufl.). Hillsdale: Erlbaum.

Cramer, C. (2019). Beruf, Rolle und Professionalität von Lehrpersonen. In E. Kiel, B. Herzig, U. Maier \& U. Sandfuchs (Hrsg.), Handbuch Unterrichten an allgemeinbildenden Schulen (S. 133-141). Stuttgart: utb.

Cramer, C., Johannmeyer, K., \& Drahmann, M. (Hrsg.). (2019). Fortbildungen von Lehrerinnen und Lehrern in Baden-Württemberg. Tübingen: Universität Tübingen.

Cramer, C., König, J., \& Grimm, M. (2020). Heimliches Curriculum in der Lehrerinnen- und Lehrerbildung. In C. Cramer, J. König, M. Rothland \& S. Blömeke (Hrsg.), Handbuch Lehrerinnen- und Lehrerbildung (S. 770-776). Stuttgart: utb.

Darling-Hammond, L., Hyler, M.E., \& Gardner, M. (2017). Effective teacher professional development. Palo Alto: Learning Policy Institute.

Daschner, P., \& Hanisch, R. (Hrsg.). (2019). Lehrkräftefortbildung in Deutschland. Weinheim: Beltz Juventa.

Desimone, L.M. (2009). Improving impact studies of teachers' professional development: toward better conceptualizations and measures. Educational Researcher, 38(3), 181-199. 
Eccles, J.S. (2005). Subjective task value and the Eccles et al. model of achievement-related choices. In A. J. Elliot \& C. S. Dweck (Hrsg.), Handbook of competence and motivation (S. 105-121). New York: Guilford.

Eid, M., Gollwitzer, M., \& Schmitt, M. (2015). Statistik und Forschungsmethoden (4. Aufl.). Weinheim: Beltz.

Fabel-Lamla, M. (2018). Der (berufs-)biographische Professionsansatz zum Lehrerberuf. In J. Böhme, C. Cramer \& C. Bressler (Hrsg.), Erziehungswissenschaft und Lehrerbildung im Widerstreit!? (S. 82-100). Bad Heilbrunn: Klinkhardt.

Garet, M.S., Porter, A.C., Desimone, L., Birman, B.F., \& Yoon, K. S. (2001). What makes professional development effective? American Educational Research Journal, 38(4), 915-945.

Gargarina, L., \& von Saldern, M. (2010). Professionalisierung der Lehrkräfte. In M. Demmer \& M. von Saldern (Hrsg.), „Helden des Alltags“. Erste Ergebnisse der Schulleitungs- und Lehrkräftebefragung (TALIS) in Deutschland (S. 49-63). Münster: Waxmann.

Gorozidis, G., \& Papaioannou, A. G. (2014). Teachers' motivation to participate in training and to implement innovations. Teaching and Teacher Education, 39, 1-11.

Graham, J. W. (2009). Missing data analysis: making it work in the real world. Annual Review of Psychology, 60(1), 549-576.

Haenisch, H. (1992). Lehrerarbeit und Lehrerfortbildung. Soest: Soester Verlagskontor.

Hayes, A. F., \& Krippendorff, K. (2007). Answering the call for a standard reliability measure for coding data. Communication Methods and Measures, 1(1), 77-89.

Hoffmann, L., \& Richter, D. (2016). Aspekte der Aus- und Fortbildung von Deutsch- und Englischlehrkräften im Ländervergleich. In P. Stanat, K. Böhme, S. Schipolowski \& N. Haag (Hrsg.), IQB-Bildungstrend 2015 (S. 481-507). Münster: Waxmann.

Jäger, R. S., \& Bodensohn, R. (2007). Bericht zur Befragung von Mathematiklehrkräften: Die Situation der Lehrerfortbildung im Fach Mathematik aus Sicht der Lehrkräfte. Bonn: Deutsche Telekom Stiftung.

Jesch, H., \& Keil, U. (2009). Wirkungen des Akkreditierungsverfahrens - Ergebnisse einer Evaluation im Kontext der Neuregelung der hessischen Lehrerfortbildung. Wiesbaden: Institut für Qualitätsentwicklung.

Johannmeyer, K., Cramer, C., \& Dobmeier, F. (2019a). Die Perspektive der Verantwortlichen der Fortbildung von Lehrerinnen und Lehrern: Ergebnisse aus Experteninterviews. In C. Cramer, K. Johannmeyer \& M. Drahmann (Hrsg.), Fortbildungen von Lehrerinnen und Lehrern in Baden-Württemberg (S. 27-40). Tübingen: Universität Tübingen.

Johannmeyer, K., Cramer, C., \& Drahmann, M. (2019b). Das Angebot an Fortbildungen für Lehrerinnen und Lehrer: Ergebnisse einer Dokumentenanalyse. In C. Cramer, K. Johannmeyer \& M. Drahmann (Hrsg.), Fortbildungen von Lehrerinnen und Lehrern in Baden-Württemberg (S. 41-52). Tübingen: Universität Tübingen.

Johannmeyer, K., Cramer, C., \& Drahmann, M. (2019c). Die Einschätzung der Teilnehmenden an Fortbildungen für Lehrerinnen und Lehrer. In C. Cramer, K. Johannmeyer \& M. Drahmann (Hrsg.), Fortbildungen von Lehrerinnen und Lehrern in Baden-Württemberg (S. 53-68). Tübingen: Universität Tübingen.

Kao, C.-P., Wu, Y.-T., \& Tsai, C.-C. (2011). Elementary school teachers' motivation toward web-based professional development, and the relationship with Internet self-efficacy and belief about web-based learning. Teaching and Teacher Education, 27(2), 406-415.

Käpplinger, B. (2011). Methodische Innovationen durch neue Nutzungen und Kombinationen einer alten Methode: das Beispiel der Programmanalyse. REPORT-Zeitschrift für Weiterbildungsforschung, 34(1), 36-44.

Krippendorff, K. (2019). Content analysis. An introduction to its methodology (4. Aufl.). Los Angeles: SAGE.

Kuckartz, U. (2018). Qualitative Inhaltsanalyse (4. Aufl.). Weinheim: Beltz Juventa.

Kunter, M., Baumert, J., Blum, W., Klusmann, U., Krauss, S., \& Neubrand, M. (2011). Professionelle Kompetenz von Lehrkräften: Ergebnisse des Forschungsprogramms COACTIV. Münster: Waxmann.

Lipowsky, F. (2010). Lernen im Beruf - Empirische Befunde zur Wirksamkeit von Lehrerfortbildung. In F. H. Müller (Hrsg.), Lehrerinnen und Lehrer lernen (S. 51-70). Münster: Waxmann.

Lipowsky, F. (2014). Theoretische Perspektiven und Empirische Befunde zur Wirksamkeit von Lehrerfortund -weiterbildung. In E. Terhart, H. Bennewitz \& M. Rothland (Hrsg.), Handbuch der Forschung zum Lehrerberuf (2. Aufl., S. 511-541). Münster: Waxmann.

Lüdtke, O., Robitzsch, A., Trautwein, U., \& Köller, O. (2007). Umgang mit fehlenden Werten in der psychologischen Forschung. Psychologische Rundschau, 58(2), 103-117. 
Ministerium für Kultus, Jugend und Sport Baden-Württemberg (2017). Ergebnisse der Befragung zur Lehrerfortbildung. Pressemitteilung vom 03.08.2017.

Neuendorf, K. A. (2002). The content analysis guidebook. Thousand Oaks: SAGE.

Nolda, S. (2018). Programmanalyse in der Erwachsenenbildung/Weiterbildung - Methoden und Forschungen. In R. Tippelt \& A. von Hippel (Hrsg.), Handbuch Erwachsenenbildung/Weiterbildung (S. 433-449). Wiesbaden: Springer VS.

OECD (2009). Creating effective teaching and learning environments. First results from TALIS. Paris: OECD Publishing.

OECD (2014). TALIS 2013 results: an international perspective on teaching and learning. Paris: OECD Publishing.

Pant, H. A., Stanat, P., Schroeders, U., Roppelt, A., Siegle, T., \& Pöhlmann, C. (2013). IQB-Ländervergleich 2012. Münster: Waxmann.

Pasternack, P., Baumgarth, B., Burkhardt, A., Paschke, S., \& Thielemann, N. (2017). Drei Phasen. Die Debatte zur Qualitätsentwicklung in der Lehrer_innenbildung. Bielefeld: Bertelsmann.

R Core Team (2019). R: A language and environment for statistical computing. Wien: R Foundation for Statistical Computing. https://www.R-project.org/. Zugegriffen: 29. Mai 2020.

Richter, D. (2011). Lernen im Beruf. In M. Kunter, J. Baumert, W. Blum, U. Klusmann, S. Krauss \& M. Neubrand (Hrsg.), Professionelle Kompetenz von Lehrkräften: Ergebnisse des Forschungsprogramms COACTIV (S. 317-325). Münster: Waxmann.

Richter, D. (2016). Lehrerinnen und Lehrer lernen: Fort- und Weiterbildung im Lehrerberuf. In M. Rothland (Hrsg.), Beruf Lehrer/Lehrerin (S. 245-260). Stuttgart: utb.

Richter, A., \& Vigerske, S. (2011). Fort- und Weiterbildung von Lehrerinnen und Lehrern - Die Bedeutung der dritten Phase am Beispiel einer Evaluation der Lehrer/-innenfortbildung im Land BadenWürttemberg.bwp @Spezial, 5, 1-15.

Richter, D., Kunter, M., Klusmann, U., Lüdtke, O., \& Baumert, J. (2011). Professional development across the teaching career: teachers' uptake of formal and informal learning opportunities. Teaching and Teacher Education, 27(1), 116-126.

Richter, D., Engelbert, M., Weirich, S., \& Pant, H. A. (2013). Differentielle Teilnahme an Lehrerfortbildungen und deren Zusammenhang mit professionsbezogenen Merkmalen von Lehrkräften. Zeitschrift für Pädagogische Psychologie, 27(3), 193-207.

Richter, E., Richter, D., \& Marx, A. (2018). Was hindert Lehrkräfte an Fortbildungen teilzunehmen? Zeitschrift für Erziehungswissenschaft, 21(5), 1021-1043.

Richter, D., Kleinknecht, M., \& Gröschner, A. (2019). What motivates teachers to participate in professional development? An empirical investigation of motivational orientations and the uptake of formal learning opportunities. Teaching and Teacher Education, 86, 102929.

Richter, E., Marx, A., Huang, Y., \& Richter, D. (2020). Zeiten zum beruflichen Lernen: Eine empirische Untersuchung zum Zeitpunkt und der Dauer von Fortbildungsangeboten für Lehrkräfte. Zeitschrift für Erziehungswissenschaft, 23(1), 145-173.

Rosseel, Y. (2012). lavaan: an R package for structural equation modeling. Journal of Statistical Software, 48(2), 1-36.

Rzejak, D., Künsting, J., Lipowsky, F., Fischer, E., Dezhgahi, U., \& Reichart, A. (2014). Facetten der Lehrerfortbildungsmotivation - eine faktorenanalytische Betrachtung. Journal for Educational Research Online, 6(1), 139-159.

Schmidt-Lauff, S. (2008). Zeit für Bildung im Erwachsenenalter. Münster: Waxmann.

Schmidt-Lauff, S. (2018). Zeittheoretische Implikationen in der Erwachsenenbildung. In R. Tippelt \& A. von Hippel (Hrsg.), Handbuch Erwachsenenbildung/Weiterbildung (S. 319-338). Wiesbaden: Springer.

Schneider, H. (2009). Nachweis und Behandlung von Multikollinearität. In S. Albers, D. Klapper, U. Konradt, A. Walter \& J. Wolf (Hrsg.), Methodik der empirischen Forschung (S. 221-236). Wiesbaden: Springer.

Schrader, J. (2011). Struktur und Wandel der Weiterbildung. Bielefeld: Bertelsmann.

Schrader, J., \& Ioannidou, A. (2011). Ziele, Inhalte und Strukturen der Erwachsenenbildung im Spiegel von Programmanalysen. In T. Fuhr, P. Gonon \& C. Hof (Hrsg.), Handbuch der Erziehungswissenschaft (S. 259-269). Paderborn: Schöningh.

Schultis, T., Holzäpfel, L., \& Leuders, T. (2014). Wirksamkeit einer Fortbildung zum produktiven Üben im Mathematikunterricht. In J. Roth \& J. Ames (Hrsg.), Beiträge zum Mathematikunterricht 2014 (S. 1111-1114). Münster: WTM. 
Stanat, P., Böhme, K., \& Schipolowski, S. (2016). IQB-Bildungstrend 2015: Sprachliche Kompetenzen am Ende der 9. Jahrgangsstufe im zweiten Ländervergleich. Münster: Waxmann.

Wolf, W., Göbel-Lehnert, U., \& Chroust, P. (1997). Lehrerfortbildung in Hessen. Marburg: Hessisches Institut für Lehrerbildung.

Yoon, K.S., Duncan, T., Lee, S. W.-Y., Scarloss, B., \& Shapley, K. (2007). Reviewing the ecidence on how teacher professional development affects student achievement. In REL 2007. Issues \& Answers Report, Bd. 033. Washington: U.S. Department of Education. 